\title{
Impact of the treatment with praziquantel and cisplatin on some biochemical parameters after Schistosoma mansoni infection and Ehrlich ascetic carcinoma in mouse model
}

\author{
Afnan El-Gowily ${ }^{1}$, Afrah Salama ${ }^{2}$ and Mohamed L. Salem ${ }^{1}$ \\ ${ }^{1}$ Chemistry Department, Faculty of Science, Tanta University, Egypt \\ ${ }^{2}$ Department of Zoology, Faculty of Science, Tanta University, Egypt
}

\section{III}

Background: Infections with Schistosoma mansoni considers a potential factor for developing cancers in different parts of the human body. Aim: This study evaluates the efficacy of cisplatin and its possible role as a complementary medicine with the anti-schistosomal drug; PZQ. Materials and Methods: Mice were infected with (40-50) S. mansoni cercariae then, after 35 days of infection, mice were (i.p.) injected with 1x106cells/mouse of EAC cells. Mice groups were divided as following; GP I (control), GP II (S. mansoni infected alone), GP III (inoculated EAC alone), GP IV (S. mansoni/EAC alone), GP V (S. mansoni/EAC treated with PZQ), GP VI (S. mansoni/EAC treated with cisplatin10 $\mu \mathrm{g}$ ), GP VII (S.

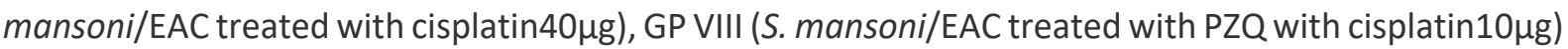
and GP IX (S. mansoni/EAC treated with PZQ with cisplatin40 $\mu \mathrm{g}$ ). Worm burden, eggs were counted. Also, tumor volume, total tumor cell counts were measured. We assessed liver and kidney function tests, otherwise we evaluate oxidative and antioxidant parameters. Results: Our data showed worm burden and egg counts were decreased in single cisplatin treated groups and the tumor cell counts in cotreated groups. Also, Hepatic GSH, GST were improved in co treated groups with PZQ than single ones, while hepatic toxicity parameters were decreased. Overall, we concluded that cisplatin even at low doses has anti-schistosomal effects. Also, PZQ co-administration with cisplatin doses ameliorated the level of GSH, it involves the maintenance of antioxidant capacity in protecting the hepatic tissue against oxidative stress by scavenging the very reactive hydroxyl and peroxyl radicals. Conclusion: Treatment with praziquantel and cisplatin alters biochemical parameters after Schistosoma mansoni infection.

Keywords: Antioxidant; Cisplatin; Ehrlichs sscites carcinoma; Praziquantel; Schistosomiasis

Editor-in-Chief: Prof. M.L. Salem, PhD - Article DOI: 10.21608/JCBR.2021.61969.1173 